\title{
Circle Location by Moments
}

\author{
Vivian Cho ${ }^{1}$ and Wm. Douglas Withers ${ }^{1,2, \star}$ \\ 1 Department of Mathematics \\ United States Naval Academy \\ 2 Accusoft Pegasus \\ Department of Mathematics, \\ United States Naval Academy, Annapolis, MD 21402 \\ wdw@usna.edu
}

\begin{abstract}
We present a method for locating circles in images based on the moment values of specially-constructed functions. Our approach differs from Hough-transform-type approaches in requiring no large accumulator array, searching operation, nor preliminary edge-detection step. It differs from previous work on curve location by moments by the existence of an explicit formula for the circle parameters and a theoretically proven guarantee of success. The moment approach is inherently robust with respect to blurriness, pixelization, or small-scale noise.
\end{abstract}

\section{Introduction}

Locating circles within images is an important computer-vision task with practical applications in astrogation, image registration, camera calibration, biometric identification, and localization of machine parts. Many approaches proposed for circle location rely on the basic approach of the Hough transform, which, though originally conceived as a line-location method, was applied to the problem of locating more general curves as early as 1972 (see [1]).
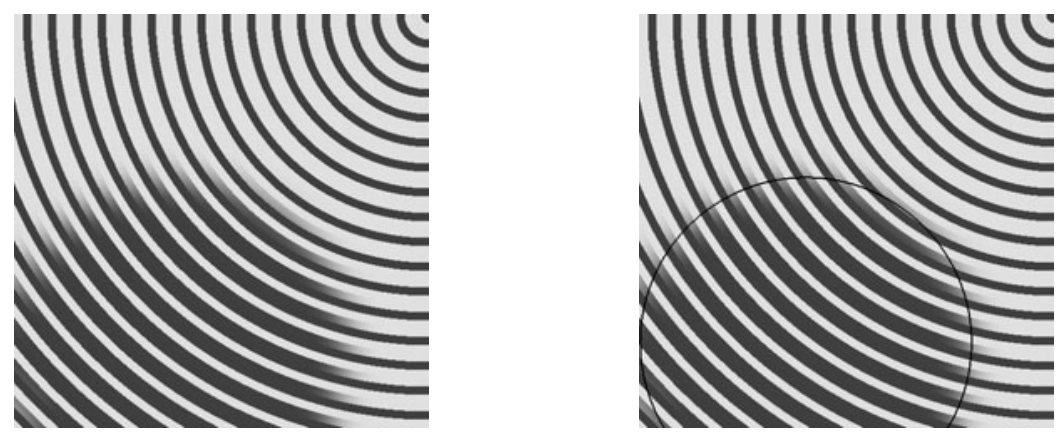

Fig. 1. Moment methods of curve location tend to seek out the single most prominent low-frequency structure

\footnotetext{
^ Corresponding author.
}

A. Elmoataz et al. (Eds.): ICISP 2010, LNCS 6134, pp. 94-102, 2010.

(C) Springer-Verlag Berlin Heidelberg 2010 
While intuitive and robust, the Hough transform presents serious resource issues of both time and space with increasing number of parameters in the family of curves under consideration. For this reason, circle-location methods using the Hough transform generally rely on one or more ingenuities to reduce the dimensionality of the required accumulator array and/or the time required to search the array; 4 includes a brief survey.

An alternative approach of using moments to locate features in images has origins as early as 1989 for the case of straight lines (see 2]). 3] presents a computationally simpler and more general method, and 4 uses moments to locate more general curves. The moment approach offers strengths complementary to those of Hough-type methods: no accumulator array is needed, no searching is involved, and the moment calculations are inherently robust with respect to blurriness and high-frequency noise. A major distinction is that while Hough-type methods yield many curves or fragments of curves within the region analyzed, moment methods yield a single global solution representing the dominant feature in the region analyzed (Fig. 11). Useful preprocessing operations and recursivesubdivision approaches to the problems of image clutter and multiple curves are discussed in [4].

The theory developed in [4] concerns a curve described by an equation

$$
\sum_{n=1}^{N} p_{n} z_{n}(x, y)=0 .
$$

This includes, for example, the case of a general circle with four-term equation:

$$
p_{1}+p_{2} x+p_{3} y+p_{4}\left(x^{2}+y^{2}\right)=0 .
$$

The method takes $S$ to be a bounded subset of the plane corresponding to an image or a subregion thereof. Let $I(x, y)$ represent the grayscale values of the image for $(x, y) \in S$. Suppose the image takes constant values on $S \cap \Gamma$ and $S-\Gamma$, where the curve $\partial \Gamma$ is described by Equation (1). Our goal is to recover the values of $p_{n}$ from moment values of the image. For a given function $U(x, y)$ defined on $S$, define the associated moment value $\langle I, U\rangle$ :

$$
\langle I, U\rangle=\iint_{S} I(x, y) U(x, y) d x d y
$$

The chief tool of the method is the following theorem:

Theorem 1. Let $V$ be an open set containing $S$. Define $s(x, y)$ twice continuously differentiable on $V$ and $F(x, y)$ continuously differentiable on $V$ such that $F(x, y)=0$ on $\partial S$. For $n=1, \ldots, N$, define $U_{n}(x, y)=\Upsilon\left(F, s, z_{n}\right)$, where

$$
\Upsilon(F, s, z)=\left(\left[s z_{y}-z s_{y}\right] F\right)_{x}-\left(\left[s z_{x}-z s_{x}\right] F\right)_{y},
$$

the $x$ and $y$ subscripts indicating partial differentiation. Then

$$
\sum_{n=1}^{N}\left\langle I, U_{n}\right\rangle p_{n}=0 .
$$


Example: For the circle, we have

$$
z_{1}(x, y)=1, z_{2}(x, y)=x, z_{3}(x, y)=y, z_{4}(x, y)=x^{2}+y^{2} .
$$

Take $S$ to be a subsquare of the image. By a change of coordinates, if necessary, we may consider $S$ to be the region $-1 \leq x \leq 1,-1 \leq y \leq 1$. Define

$$
F(x, y)=\max \left(0,1-x^{2}\right) \max \left(0,1-y^{2}\right),
$$

then $F \equiv 0$ on $\partial S$ as required by Theorem 1 . Define $s(x, y) \equiv 1$. Then we have:

$$
\begin{aligned}
& U_{1}(x, y)=\Upsilon(F, s, 1)=0 \\
& U_{2}(x, y)=\Upsilon(F, s, x)=2 y\left(1-x^{2}\right) \\
& U_{3}(x, y)=\Upsilon(F, s, y)=-2 x\left(1-y^{2}\right) \\
& U_{4}(x, y)=\Upsilon\left(F, s, x^{2}+y^{2}\right)=4 x y\left(y^{2}-x^{2}\right) .
\end{aligned}
$$

If we now further suppose that our image function $I(x, y)$ is zero-valued outside a circle of radius $3 / 2$ centered at $(-1 / 2,-1)$ and has value 1 inside the circle (corresponding to $p_{1}=-1, p_{2}=1, p_{3}=2, p_{4}=1$ ), we can calculate the corresponding moment values over $S$ :

$$
u_{1}=0, u_{2}=-1.13136, u_{3}=0.561783, u_{4}=0.007794 \text {. }
$$

It is easily verified that (3) holds.

Here the functions $F$ and $s$ are largely arbitrary. In practice the differentiability assumptions can be relaxed somewhat. Various choices of $(F, s)$ yield various equations of the form (3). The goal is to generate a system of such equations solvable for the parameters $p_{n}$ up to multiplication by a single nonzero constant (which leaves invariant the curve described by (10)).

In practice $F$ also serves to define the region $S$, as the region where $F(x, y)$ is nonzero together with its boundary. In this article we take $F$ as defined by (4) so that $S$ is the square $[-1,1] \times[-1,1]$. With appropriately defined coordinates, this square can represent any subsquare of the image.

Theorem 1 leaves us with two problems. The first is choosing a set of $(F, s)$ yielding a system of equations solvable for $p_{n}$, the ease or difficulty of which is highly dependent on the class of curves under consideration. The bare minimum number of equations required is the number of independent parameters in the curve equation. Not infrequently more are needed, due to redundancy of one or more equations - which may, moreover, be contingent upon the curve position.

The second problem is solving the resulting system of equations - a thoroughly understood but possibly complicated matter of linear algebra. Explicit formulas for the solution of the system are therefore valuable if available.

Two examples: (1) The relatively simple case of locating straight lines ([3]) uses three equations based on three distinct moment functions, with a simple explicit form for the solution. (2) The more complex problem of locating a general conic with five parameters uses 18 equations based on 45 distinct moment functions in order to minimize the potential for excess redundancy; however, for 
the class of general conic curves this potential persists for fundamental reasons of geometry no matter how many equations are used. Special measures for coping with this and other aspects of this problem are discussed at length in 4.

This article treats the family of circles described by (2) and presents a method based on Theorem 1 for locating circles in images using moment information. This class of curves is a subset of that treated in [4] we use the special properties of this class to present a specialized solution, which is simpler to implement than the general approach of [4]. Moreover, in contrast to [4, we give precise conditions guaranteeing the existence of a unique solution and an explicit formula for the solution.

\section{Theory}

A circle with center $\left(x_{0}, y_{0}\right)$ and radius $r$ has equation (2), where $p_{4} \neq 0$ and

$$
p_{2}=-2 p_{4} x_{0}, p_{3}=-2 p_{4} y_{0}, p_{1}=p_{4}\left(x_{0}^{2}+y_{0}^{2}-r^{2}\right) .
$$

At least one of $p_{2}, p_{3}, p_{4}$ must be nonzero for (2) to describe a curve. In the case $p_{4}=0$, this equation describes a straight line, which is a limiting case of a circle as the radius goes to infinity. Our method locates straight lines or circles with a unified approach. We use the term "generalized circle" to refer to either a circle or straight line, as opposed to a "true circle" with finite radius $\left(p_{4} \neq 0\right)$.

Perhaps the simplest conceivable method would be to define moment functions $M_{n}$ such that $\left\langle I, M_{n}\right\rangle=p_{n}$. Unfortunately, no such set of functions exists. For necessarily $\left\langle I, M_{4}\right\rangle=0$ for any $I$ consisting of a single straight edge $\left(p_{4}=0\right)$. One can then argue, using the invertibility of either the Radon or Fourier transform, that $M_{4} \equiv 0$, making recovery of $p_{4}$ impossible.

Our slightly more complicated method operates in two phases. The first phase identifies a line $\Lambda$ with equation

$$
x \cos \phi+y \sin \phi=\kappa
$$

which intersects $\partial \Gamma$ orthogonally. The second phase defines a set of moment functions $M_{1} \ldots M_{4}$ in terms of $\Lambda$ such that $\left\langle I, M_{n}\right\rangle=p_{n}$ for any generalized circle orthogonal to $\Lambda$.

Phase I: Determining an Orthogonal Line. Let $I(x, y), S, V$, and $F(x, y)$ be as in Theorem 1, and assume $\partial \Gamma$ is a generalized circle described by (1). Applying Theorem 1 with $s=1$, define:

$$
\begin{aligned}
& A(x, y)=\Upsilon(F, 1, x)=-F_{y} \\
& B(x, y)=\Upsilon(F, 1, y)=F_{x} \\
& C(x, y)=\Upsilon\left(F, 1, x^{2}+y^{2}\right)=2 y F_{x}-2 x F_{y} .
\end{aligned}
$$

Note $\Upsilon(F, 1,1)=0$. Theorem 1 then yields:

$$
\langle I, A\rangle p_{2}+\langle I, B\rangle p_{3}+\langle I, C\rangle p_{4}=0 .
$$


We claim that (6) describes a line orthogonal to $\partial \Gamma$ if $\rho, \phi, \kappa$ satisfy:

$$
\langle I, A\rangle=\rho \cos \phi,\langle I, B\rangle=\rho \sin \phi,\langle I, C\rangle=2 \rho \kappa .
$$

For true circles it suffices to show that the center $\left(x_{0}, y_{0}\right)$ lies on $\Lambda$. This is verifiable by direct substitution of (5) and (8) into (7). In the straight-line case orthogonality follows from the value $\langle I, B\rangle /\langle I, A\rangle$ of the slope of $\partial \Gamma$ (see [3]).

This method fails if $\langle I, A\rangle=\langle I, B\rangle=\rho=0$. In the straight-line case (see [3]) $\rho>0$ provided that $F(x, y) \geq 0$ everywhere (nonnegativity) and $\partial \Gamma$ intersects the support of $F$ nontrivially (impingency). Impingency is an obvious requirement; a curve disjoint from the set of pixels to be examined is unlocatable For the true-circle case we provide the following back-up method. For the sake of brevity we merely sketch the derivation.

An alternate formulation for $\Lambda$, and $\kappa$ in particular, is given by defining:

$$
P(x, y)=\Upsilon(F, x, y), Q(x, y)=\Upsilon\left(F, x, x^{2}+y^{2}\right), R(x, y)=\Upsilon\left(F, y, x^{2}+y^{2}\right) .
$$

Let $\rho, \phi$ be defined as in (8). Then it can be shown that:

$$
x_{0}=\frac{p_{1} \rho \sin \phi}{2\langle I, P\rangle p_{4}}-\frac{\langle I, R\rangle}{2\langle I, P\rangle}, y_{0}=-\frac{p_{1} \rho \cos \phi}{2\langle I, P\rangle p_{4}}+\frac{\langle I, Q\rangle}{2\langle I, P\rangle} .
$$

It follows that

$$
x_{0} \cos \phi+y_{0} \sin \phi=\frac{\langle I, Q\rangle \sin \phi-\langle I, R\rangle \cos \phi}{2\langle I, P\rangle}=\kappa \text { (alternate). }
$$

Formula (9) also fails if $\langle I, P\rangle=0$; however, it can be proven that nonnegativity and impingency together guarantee that at least one of $\rho$ or $\langle I, P\rangle$ is nonzero.

This alternate formulation of $\Lambda$ is numerically stable as $\rho \rightarrow 0$ in the following subtle sense: even though $\phi$ as defined by (8) — and hence the position of $\Lambda$ - is not numerically stable, the (ideally zero) distance from $\left(x_{0}, y_{0}\right)$ to $\Lambda$ is increasingly insensitive to errors in $\phi$ as $\rho \rightarrow 0$. For example, in the extreme case $\rho=0$,

$$
x_{0}=-\frac{\langle I, R\rangle}{2\langle I, P\rangle}, y_{0}=\frac{\langle I, Q\rangle}{2\langle I, P\rangle},
$$

so that (9) holds for any value of $\phi$.

Phase II: Determining the Circle Parameters. Now assume $\partial \Gamma$ to be defined by Equation (2) and $\Lambda$ to be a line with equation (6) (determined by the method of the previous section or any other) which intersects $\partial \Gamma$ orthogonally. Let $S$ and $V$ be sets satisfying the conditions of Theorem 1 , and let $G(x, y)$ be a function satisfying the same conditions as $F(x, y)$ in Theorem 1. $G$ need not be (and in fact should not be, for reason given below) the same as $F$ used in Phase I. The parameters of Equation (2) can then be determined as follows:

Theorem 2. Define:

$$
\begin{aligned}
& M_{1}(x, y)=\Upsilon\left(G,-x \sin \phi+y \cos \phi,-2 \kappa[x \cos \phi+y \sin \phi]+x^{2}+y^{2}\right) ; \\
& M_{2}(x, y)=\Upsilon\left(G, 1,-2 \kappa y+\left(x^{2}+y^{2}\right) \sin \phi\right) ; \\
& M_{3}(x, y)=\Upsilon\left(G, 1,2 \kappa x-\left(x^{2}+y^{2}\right) \cos \phi\right) ; \\
& M_{4}(x, y)=\Upsilon(G, 1,-x \sin \phi+y \cos \phi) .
\end{aligned}
$$


Then all points $(x, y)$ on $\partial \Gamma$ satisfy

$$
\left\langle I, M_{1}\right\rangle+\left\langle I, M_{2}\right\rangle x+\left\langle I, M_{3}\right\rangle y+\left\langle I, M_{4}\right\rangle\left(x^{2}+y^{2}\right)=0 .
$$

Proof. This is a matter of direct calculation, aided by Green's Theorem, which yields:

$$
\begin{aligned}
\langle I, \Upsilon(G, s, z)\rangle & =h \iint_{\Gamma} \Upsilon(G, s, z) d x d y \\
& =h \oint_{\partial \Gamma} G\left(\left[s z_{x}-z s_{x}\right] d x+\left[s z_{y}-z s_{y}\right] d y\right)
\end{aligned}
$$

where $h$ is the difference in gray-levels between the left and right sides of $\partial \Gamma$. Note in particular:

$$
\iint_{\Gamma} \Upsilon(G, 1, z) d x d y=h \oint_{\partial \Gamma} G d z
$$

Even in the straight-line case, $\partial \Gamma$ can be treated as a closed curve by assuming it bends around outside the region $S$ (where $G$ is zero-valued).

For example, we have:

$$
\left\langle I, M_{4}\right\rangle=h \oint_{\partial \Gamma} G d(-x \sin \phi+y \cos \phi) .
$$

Consider first the true-circle case. Following (5), it suffices to show:

$$
\begin{aligned}
& \left\langle I, M_{1}\right\rangle=\left(x_{0}^{2}+y_{0}^{2}-r^{2}\right)\left\langle I, M_{4}\right\rangle ; \\
& \left\langle I, M_{2}\right\rangle=-2 x_{0}\left\langle I, M_{4}\right\rangle ; \\
& \left\langle I, M_{3}\right\rangle=-2 y_{0}\left\langle I, M_{4}\right\rangle .
\end{aligned}
$$

Introducing a parametric representation for the the circle:

$$
x=x_{0}+r \cos \theta, y=y_{0}+r \sin \theta, 0 \leq \theta \leq 2 \pi,
$$

we find:

$$
\left\langle I, M_{4}\right\rangle=h \oint_{\partial \Gamma} G(r \sin \theta \sin \phi+r \cos \theta \cos \phi) d \theta=h \oint_{\partial \Gamma} G r \cos (\theta-\phi) d \theta .
$$

We then calculate:

$$
\begin{aligned}
\left\langle I, M_{3}\right\rangle & =h \oint_{\partial \Gamma} G d\left(2 \kappa x-\left(x^{2}+y^{2}\right) \cos \phi\right) \\
& =h \oint_{\partial \Gamma} G\left(-2 \kappa r \sin \theta+2 x_{0} r \cos \phi \sin \theta-2 y_{0} r \cos \theta \cos \phi\right) d \theta \\
& =h \oint_{\partial \Gamma} G\left(-2 r \sin \theta\left[\kappa-x_{0} \cos \phi\right]-2 y_{0} r \cos \theta \cos \phi\right) d \theta \\
& =h \oint_{\partial \Gamma} G\left(-2 r \sin \theta\left[y_{0} \sin \phi\right]-2 y_{0} r \cos \theta \cos \phi\right) d \theta \\
& =-2 y_{0} h \oint_{\partial \Gamma} G r \cos (\theta-\phi) d \theta=-2 y_{0}\left\langle I, M_{4}\right\rangle .
\end{aligned}
$$


$\left\langle I, M_{1}\right\rangle$ and $\left\langle I, M_{2}\right\rangle$ are treated similarly.

In the straight-line case, let $\left(x_{1}, y_{1}\right)$ be the point of intersection of $\partial \Gamma$ and $\Lambda$. Being orthogonal to $\Lambda, \partial \Gamma$ has equation $p_{1}+p_{2} x+p_{3} y=0$, where

$$
\begin{aligned}
& p_{1}=y_{1} \cos \phi-x_{1} \sin \phi, \\
& p_{2}=\sin \phi, \\
& p_{3}=-\cos \phi,
\end{aligned}
$$

(and $\left.p_{4}=0\right)$. We can also write $\partial \Gamma$ parametrically as:

$$
x=x_{1}+t \cos \phi, y=y_{1}+t \sin \phi .
$$

Direct calculation then yields $\left\langle I, M_{n}\right\rangle=K p_{n}$, where

$$
K=h \oint_{\partial \Gamma} 2 G t d t .
$$

A final concern is that (10) might hold trivially, in that $\left\langle I, U_{n}\right\rangle=0$ for all $n$. (In fact, this happens if $G$ is taken identical to $F$ from Phase I.) We can avoid this by noting that in the true-circle case (11):

$$
x \cos \phi+y \sin \phi-\kappa=r \cos (\theta-\phi),
$$

while in the straight-line case (13):

$$
x \cos \phi+y \sin \phi-\kappa=t .
$$

It therefore suffices to choose $G(x, y)$ so that $(x \cos \phi+y \sin \phi-\kappa) G(x, y)$ is nonnegative - for example:

$$
G(x, y)=(x \cos \phi+y \sin \phi-\kappa) \max \left(0,1-x^{2}\right) \max \left(0,1-y^{2}\right) .
$$

Impingency then implies that the integrand of (12) or (14) is positive somewhere and negative nowhere, so the resulting integral is necessarily nonzero.

A general discussion of the organization of the method for computational efficiency is given in [4]. As noted there, the moment computation dominates the operation count (even more so for the simplified method presented here), and the overriding concern is the degree of the moment functions in $x$ alone. The functions used in Phase I are all of cubic degree or lower in $x$; this phase of the computation thus can be implemented using three multiplications and four additions per pixel. The functions used in Phase II are all of fifth degree or lower; Phase II thus entails an additional two multiplications and two additions per pixel.

\section{$3 \quad$ Examples}

This section presents illustrations of our method in practice, with particular emphasis on the special capabilities of the moment approach. To begin with, we 

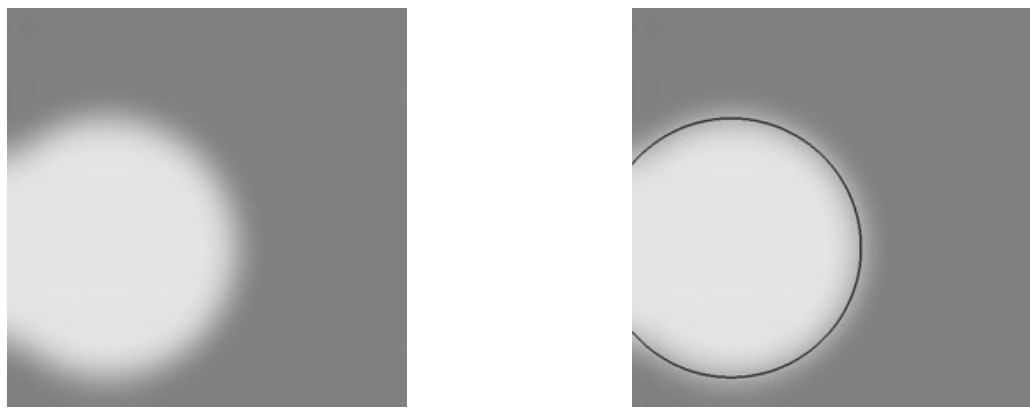

Fig. 2. Circle location on a blurred image
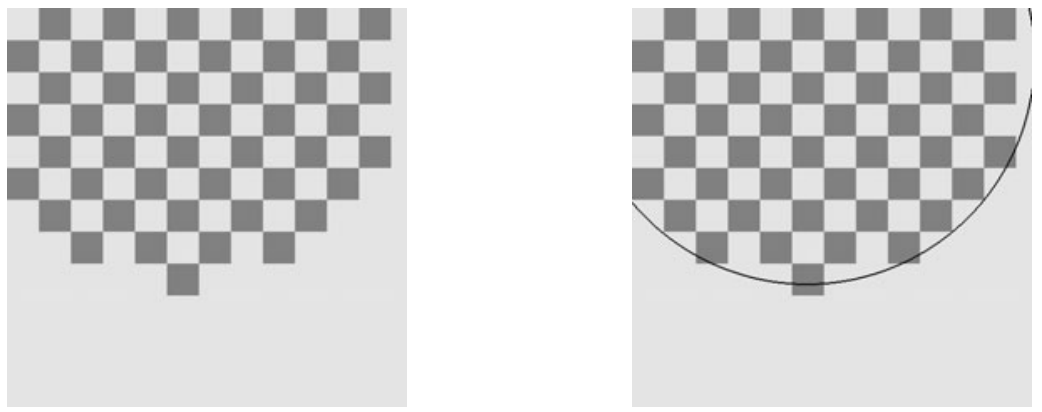

Fig. 3. Fitting a circle to an approximate boundary

ran a series of 5000 test cases on randomly-generated $500 \times 500$ images containing whole or partial circles. In all cases the circle contained between $10 \%$ and $90 \%$ of the image area. Goodness of fit was measured by reconstructing the image from the calculated parameter values and counting the pixels differing between the image and its reconstruction. The average case showed a difference in $0.0093 \%$ of pixel values. The worst case showed a difference in $1.36 \%$ of pixel values.

Figure2 shows our method used for locating a blurred circle. This type of image is problematic for Hough-type approaches or other methods requiring a preliminary edge detection step. However, the moment method, which relies on global moment values, is scarcely impeded. In this and all other examples, with the exception of Fig. 5, the method was applied with no preprocessing whatsoever.

Figure 1 shows successful location of a circle which is not only blurred but overlaid with distracting high-frequency features. Figure 3 shows fitting of a circle to an approximate circular boundary. Figure 4 shows successful circle location in an image consisting of the same two pixel values both inside and outside the circle, the boundary marked only by a shift in frequency.

Figure 5] shows the possible application of our method to the problem of locating the pupil in a red-eye-removal application. In this case simple preprocessing 

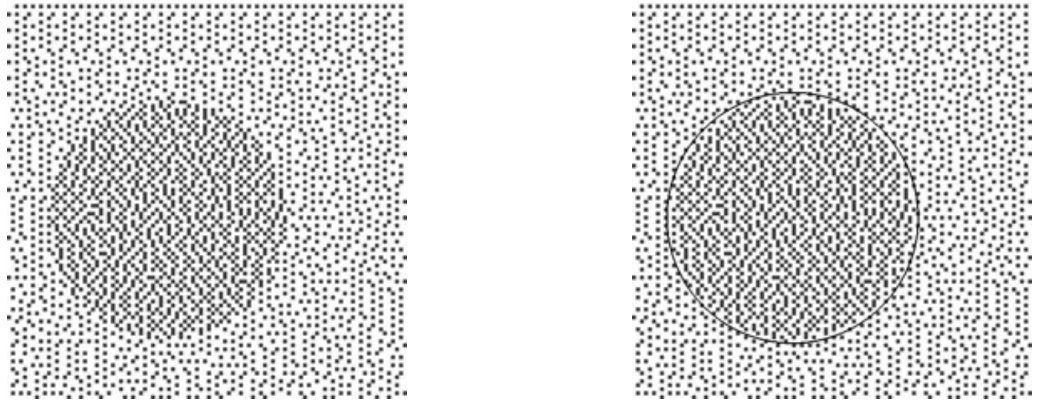

Fig. 4. Locating a circle defined by a change in frequency

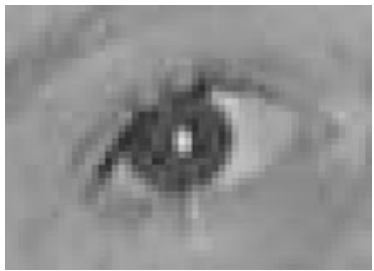

(a)

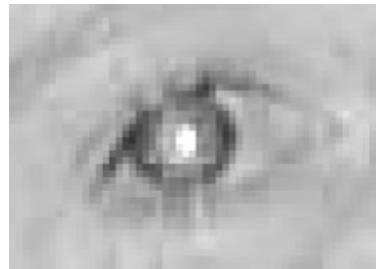

(b)

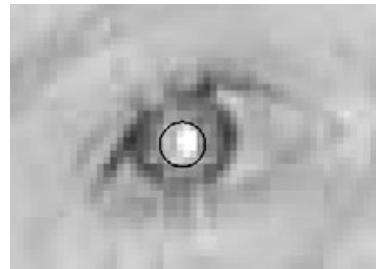

(c)

Fig. 5. Locating pupil in a potential red-eye removal application: (a) grayscale representation of the (originally color) image; (b) red component of the image, showing flare in the pupil; (c) pupil located to sub-pixel accuracy.

(paint a pixel white if its red component value exceeds its green component value by 77 or more, gray otherwise) was used to isolate the red-flare region. Subsequent application of our method serves to locate the pupil with subpixel accuracy.

\section{References}

1. Duda, R.O., Hart, P.E.: Use of the Hough Transform to Detect Lines and Curves in Pictures. Communications of the ACM 15, 11-15 (1972)

2. Lyvers, E.P., Mitchell, O.R., Akey, M.L., Reeves, A.P.: Subpixel Measurements Using a Moment-Based Edge Operator. IEEE Trans. Pattern Anal. Machine Intell. 11(12), 1293-1309 (1989)

3. Popovici, I., Withers, W.D.: Custom-Built Moments for Edge Location. IEEE Trans. Pattern Anal. and Machine Intell. 28(4), 637-642 (2006)

4. Popovici, I., Withers, W.D.: Curve Parametrization by Moments. IEEE Trans. Pattern Anal. and Machine Intell. 31(1), 15-26 (2009) 